\title{
The nutritional status of asymptomatic HIV-infected Africans: directions for dietary intervention?
}

\author{
Hester H Vorster ${ }^{1, *}$, Annamarie Kruger ${ }^{1}$, Barrie M Margetts ${ }^{2}$, Christina S Venter $^{1}$, \\ H Salomé Kruger ${ }^{1}$, Frederick J Veldman ${ }^{3}$ and Una E Maclntyre ${ }^{4}$ \\ 'School of Physiology and Nutrition, Faculty of Health Sciences, North-West University Potchefstroom Campus, \\ Private Bag X6001, Potchefstroom 2520, Republic of South Africa: ${ }^{2}$ Public Health Nutrition, Institute of Human \\ Nutrition, University of Southampton, Southampton, UK: ${ }^{3}$ Department of Paramedical Sciences, Free State \\ Technikon, Bloemfontein, Republic of South Africa: ${ }^{4}$ Department of Paediatrics, Medical University of South Africa, \\ Pretoria, Republic of South Africa
}

Submitted 6 November 2003: Accepted 21 May 2004

\begin{abstract}
Objective: To compare the relationships between food (nutrient) intakes and biochemical markers of nutritional status of asymptomatic HIV-infected with HIVuninfected subjects, to gain more information on the appropriate diet for HIV-infected persons at an early stage of infection.

Design: Cross-sectional population-based survey.

Setting: North West Province, South Africa.

Subjects: Two hundred and sixteen asymptomatic HIV-infected and 1550 HIVuninfected men and women volunteers aged 15 years and older, recruited as 'apparently healthy' subjects from 37 randomly selected sites.

Outcome measures: Food and nutrient intakes, measured with a validated foodfrequency questionnaire, and nutritional status indicated by anthropometric and biochemical variables, measured by a standardised methodology.

Results: The prevalence of HIV infection in the study population was $11.9 \%$. The anthropometric indices and nutrient intakes of HIV-infected and uninfected subjects did not differ significantly, indicating that these 216 HIV-infected subjects were at an early stage of infection. Of the biochemical nutritional status variables, high-density lipoprotein cholesterol and total cholesterol, haemoglobin, albumin and triglycerides were significantly lower in infected subjects. They also had higher globulin and liver enzyme levels than uninfected subjects. In infected subjects, serum albumin correlated significantly with serum lipids, serum vitamin A, serum vitamin E, serum iron, total iron-binding capacity and haemoglobin. The significant positive correlations of the liver enzymes with serum lipids, albumin, vitamin A and iron, observed in HIV-uninfected subjects, disappeared in the infected subjects. Polyunsaturated fat intake showed significant positive correlations with the increased liver enzymes in infected subjects. A principal components analysis indicated that, in infected subjects, increased liver enzymes correlated with higher consumption of maize meal and lower consumption of meat and vegetables.

Conclusions and recommendations: This survey indicated that asymptomatic HIVinfected subjects who followed a diet rich in animal foods had smaller decreases in serum albumin, haemoglobin and lipid variables, and smaller increases in liver enzymes, than those who consumed a diet based on staple foods. This suggests that animal foods are associated with improved nutritional status in HIV-infected persons. These results should be confirmed with intervention studies before dietary recommendations for asymptomatic HIV-infected individuals can be made.
\end{abstract}

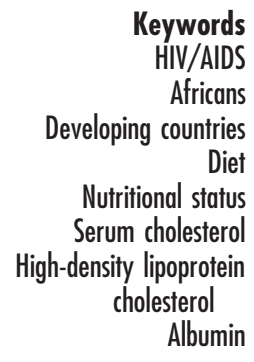

Keywords Africans Diet

Nutritional status serum cholesterol olesterol

Albumin
The global HIV/AIDS pandemic is assuming proportions in sub-Saharan African countries that are threatening to neutralise previous gains in health ${ }^{1}$, reducing life expectancy in some of these countries to less than 40 years $^{2}$, and paralysing health-care services and economies $^{3}$. In South Africa, the prevalence of HIV infection was based on anonymous testing of pregnant women attending antenatal clinics for the first time over a selected period $^{4}$. The estimated prevalence rose from $7.6 \%$ in 1994 to $10.4 \%$ in $1995,14.2 \%$ in 1996, $17.0 \%$ in 1997 and to $22.5 \%$ in $1998^{4}$. The South African HIV household survey in 2002 indicated that $11.4 \%$ of South Africans over the age 
of 2 years ( 4.5 million people) are living with HIV/AIDS 5 . In a population of just under 40 million $^{4}$, it is estimated that in 1998 alone more than 100000 children were orphaned by AIDS-related deaths ${ }^{6}$, and that by the year $2010,16 \%$ of all South African children will be orphans, more than $70 \%$ of whom will have been orphaned by $\operatorname{AIDS}^{5}$.

Due to economic constraints, in most sub-Saharan African countries anti-retroviral therapy is beyond the reach of all but a privileged $\mathrm{few}^{3}$, and in the past, emphasis was on prevention strategies. In 2003, the South African Government announced a comprehensive plan for the care, management and treatment of people infected with the virus and suffering from AIDS $^{7}$. Optimising nutritional status is a key objective in this plan ${ }^{7}$. Considering that free anti-retroviral therapy will probably not be available for some time and especially not for asymptomatic people, the role of nutrition is important at this stage of the infection. It is generally accepted that 'optimum' nutrition could improve the quality of life of people living with HIV/ AIDS, that it may slow the progression of HIV infection to AIDS and that it may improve tolerance to anti-retroviral therapy $^{8-15}$.

In rural Uganda, rates of all-cause mortality in HIV/AIDS subjects are much higher and progression times to death much shorter than in developed countries?. This could possibly be related to poor nutritional status. There is an established relationship of wasting ${ }^{10}$, micronutrient deficiencies ${ }^{11}$ and oxidative stress ${ }^{12}$ with progression of HIV/AIDS. The World Health Organization and the Food and Agriculture Organization of the United Nations recognised the importance of nutrition in people living with HIV/AIDS and produced a manual on nutritional care for infected people and caregivers ${ }^{16}$. This manual focuses on practical advice, concentrating on local available foods, beverages and spices. The manual recommends 'healthy, balanced' nutrition with seven food-based messages: enjoy a variety of foods; eat staple foods with each meal; eat legumes if possible every day; eat animal and milk products regularly; eat vegetables and fruit every day; use fats and oils as well as sugar and sugary foods; drink plenty of clean and safe water. These messages are compatible with 'adequate' intakes of energy and micronutrients and also with traditional staple foods, which will make the diet affordable and acceptable, provided that household food security is not compromised.

There are two major unanswered questions regarding nutrition of HIV-infected people. The first is whether 'optimal' nutritional status will in fact delay the progression of the disease and the second is what type of diets (foods and combination of foods) will lead to optimal nutrition status in infected people. There seems to be circumstantial evidence ${ }^{7-15}$ that the first question can be answered in the affirmative. It may be difficult to answer the second question - ethical considerations in conducting clinical trials with different diets in infected subjects may be problematic. One way to seek an indirect answer to this second question is to examine relationships between dietary/nutrient intakes and nutritional status of infected people in epidemiological studies. In this paper we report on these relationships in 216 asymptomatic HIVinfected subjects compared with 1550 uninfected subjects who participated in the THUSA survey, conducted from 1996 and 1998 in the North West Province of South Africa.

\section{Methods}

\section{Subjects and study design}

'Thusa' is a Setswana word meaning help, and is also an acronym for Transition and Health during Urbanisation of South Africans. The main aim of the THUSA survey was to monitor the impact of urbanisation on health determinants of South Africans to provide information for appropriate health interventions. The methods of sample selection, all measurements and analyses have previously been described $^{17}$. Briefly, 1854 'apparently healthy' African volunteers, aged 15 years and older, were recruited from 37 randomly selected sites throughout the North West Province. Subjects were stratified into five levels of urbanisation, based on where they lived and type of employment. For data analysis on HIV-infected and uninfected subjects, subjects were grouped together, but statistical analyses controlled for area of residence. Pregnant and lactating women, subjects with any known disease, those taking any form of chronic medication, with oral temperature above $37^{\circ} \mathrm{C}$ and inebriated subjects were excluded. Complete data were available for 1817 subjects.

\section{Measurements and biochemical analyses}

Demographic information, health histories, psychological profiles $^{18}$ and dietary intake data ${ }^{19}$ were obtained during individual interviews by specially trained, multilingual fieldworkers in the language of the subject's choice, using questionnaires specially designed or adapted and validated for this population. A validated ${ }^{20}$ quantitative foodfrequency questionnaire was used to obtain habitual dietary intake data. Nutrient intakes were calculated using a programme based on the South African food composition tables ${ }^{21}$. Anthropometric measurements (height, weight, body circumferences and skinfold thicknesses) were measured in triplicate by post-graduate biokinetics students, standardised by a level III anthropometrist. Two experienced researchers measured blood pressures after subjects have been seated for at least $10 \mathrm{~min}$. Two specially trained nursing sisters assessed clinical signs of malnutrition. Fasting blood samples were drawn from the vena cephalica using a sterile butterfly infusion set (Johnson \& Johnson; 21G, $19 \mathrm{~mm}$ ) and sterile syringes, after which a 2 hour glucose tolerance test was performed. Serum, citrated and EDTA (ethylenediaminetetraacetic acid) plasma were prepared immediately in the field using a Universal 16R ${ }^{\mathrm{TM}}$ Hettich centrifuge (Tuttlingen, Germany) with cooling facilities. Haematocrit and 
haemoglobin concentrations were determined in the field on EDTA blood. Serum and plasma samples were stored in Eppendorff tubes at $-20^{\circ} \mathrm{C}$ in the field for $2-4$ days and later at $-84^{\circ} \mathrm{C}$ in the laboratory. Serum proteins, minerals, electrolytes, glucose, lipids and enzymes were determined with the DAX system (discrete analyser, Technicon DAX48; Miles Inc. Diagnostic Division, Tarrytown, NY, USA). Serum insulin, vitamins $\mathrm{A}, \mathrm{B}_{6}, \mathrm{~B}_{12}$ and $\mathrm{E}$, iron, ferritin, transferrin and iron-binding capacity as well as erythrocyte folate and urinary sodium and potassium were determined with high-performance liquid chromatography, immunological and colorimetric methods. Plasma fibrinogen was measured with the Clauss method using the ACL 200 (Instrumentation Laboratory, Milan, Italy) system. HIV status was determined with an enzymeimmunological method (Enzymum-Test, anti-HIV $1+2+$ subtype, catalogue no. 1557319; Boehringer Mannheim, Mannheim, Germany). Appropriate standards were used in all instances.

\section{Statistical analysis}

The data were analysed using the SPSS package (SPSS Inc., Chicago, IL, USA). Means, medians, standard deviations, standard errors and 95\% confidence intervals were calculated. Data that were not normally distributed were normalised by logarithmic transformation, or where normalisation was not possible, data were analysed using non-parametric statistical tests.

Analysis of variance adjusted for age, gender, area of residence and year of study was used to compare metabolic variables of $\mathrm{HIV}$-infected and uninfected subjects. Partial correlations, controlling for age, gender, area of residence and year of study, were determined to explore associations between nutrient intakes and biochemical indicators of nutritional status. A principal components analysis was used to derive a summary measure of the relative importance of the foods measured in the food-frequency questionnaire. This summary measure was subsequently included in regression models to provide an estimate of overall dietary patterns and relationships with biochemical variables.

Data collections of the THUSA study were done in 1996 and 1998. To test if the two sets of data could be combined, total reported energy intakes of women in the 1996 and 1998 datasets were compared. The mean intake of the 1996 group was $7973.7 \mathrm{~kJ}$ and that in the 1998 group was $7997.1 \mathrm{~kJ}$, and it was judged that the two sets of data could be combined.

\section{Etbical considerations}

The study was done with the full co-operation of the North West Department of Health and Social Services and with the permission of the various communities from which subjects were recruited. It was also approved by the Ethics Committee of Potchefstroom University (approval no. 4M5-95). All subjects were fully informed about the objectives and procedures of the study in their home language and all signed an informed consent form. Illiterate people signed with a cross. After completion of the study, additional approval from the same ethics committee (but not from the subjects) was obtained to test anonymously for HIV status. Subjects identified with hypertension, diabetes mellitus and anaemia (who complained of not feeling well) were referred to local clinics, hospitals or their physicians. Subjects received lunch after completion of the glucose tolerance test and their travel expenses were paid. Volunteers who did not meet the inclusion criteria were screened for hypertension and diabetes mellitus and referred for treatment if necessary. Subjects who requested HIV testing were informed that this testing could be obtained from health facilities with pre- and post-test counselling capacity.

\section{Results}

Table 1 shows that of these 'apparently healthy' asymptomatic subjects, $12.3 \%$ of the men and $11.5 \%$ of the women tested HIV-positive. The highest rates were seen in the 25-34.9-year-old groups. There was a statistically significant increase in the percentage of infected subjects from 1996 to 1998.

Table 2 compares the mean age, anthropometric indices and blood pressures of HIV-infected and uninfected men and women. The HIV-infected subjects were slightly but significantly younger than the uninfected subjects. When adjusted for age, gender, area of residence and year of

Table 1 Proportion of HIV-infected men and women by year of study and age group

\begin{tabular}{lc}
\hline $\begin{array}{l}\text { Gender, year and } \\
\text { age group }\end{array}$ & $\begin{array}{c}\text { Percentage } \\
(95 \% \text { confidence interval) }\end{array}$ \\
\hline Men & \\
Both years & $12.3(10.2-14.8)$ \\
1996 & $8.8(6.2-12.3)^{*}$ \\
1998 & $15.2(12.2-18.9)^{\star}$ \\
Age group (years) & \\
$15-24.9$ & 11.9 \\
$25-34.9$ & 16.9 \\
$35-44.9$ & 12.9 \\
$45-54.9$ & 7.8 \\
$55-64.9$ & 14.7 \\
$65+$ & 7.3 \\
Women & \\
Both years & $11.5(9.7-13.6)$ \\
1996 & $8.1(6.0-10.8)^{\star}$ \\
1998 & $14.8(12.1-18.0)^{*}$ \\
Age group (years) & \\
$15-24.9$ & 13.9 \\
$25-34.9$ & 14.7 \\
$35-44.9$ & 10.7 \\
$45-54.9$ & 8.4 \\
$55-64.9$ & 7.6 \\
$65+$ & 11.9 \\
\hline
\end{tabular}

Adjusted for age, area of residence and year of study. * Significantly different $(P \leq 0.05)$. 
Table 2 Comparison of mean (standard deviation) age, anthropometry and blood pressure of HIV-infected and uninfected men and women

\begin{tabular}{|c|c|c|c|c|c|}
\hline \multirow[b]{2}{*}{ Variable } & \multicolumn{2}{|c|}{ Men } & \multicolumn{2}{|c|}{ Women } & \multirow[b]{2}{*}{ Significance ${ }^{*} \dagger$} \\
\hline & $\begin{array}{l}\text { HIV-infected } \\
\quad(n=97)\end{array}$ & $\begin{array}{l}\text { HIV-uninfected } \\
\quad(n=654)\end{array}$ & $\begin{array}{l}\text { HIV-infected } \\
(n=119)\end{array}$ & $\begin{array}{l}\text { HIV-uninfected } \\
\quad(n=896)\end{array}$ & \\
\hline Age (years) & $35.1(14.5)$ & $37.7(15.5)$ & $34.8(12.7)$ & $38.2(14.3)$ & $0.002 \dagger$ \\
\hline $95 \%$ confidence interval & $32.2-38.1$ & $35.5-38.9$ & $32.5-37.1$ & $37.3-39.1$ & \\
\hline Body mass index $\left(\mathrm{kg} \mathrm{m}^{-2}\right)$ & $20.1(3.1)$ & $21.2(4.2)$ & $26.1(5.6)$ & $27.0(6.9)$ & $\mathrm{NS}^{*}$ \\
\hline Sum of seven skinfold thicknesses $(\mathrm{mm})$ & $64.8(33.4)$ & $70.7(42.0)$ & $115.1(99.2)$ & $96.0(86.6)$ & $\mathrm{NS}^{*}$ \\
\hline Waist-to-hip ratio & $0.85(0.09)$ & $0.84(0.06)$ & $0.76(0.07)$ & $0.76(0.07)$ & \\
\hline Systolic blood pressure (mmHg) & $127.4(16.7)$ & $126.6(16.8)$ & $122.1(16.6)$ & $128.0(22.2)$ & $\mathrm{NS}^{*}$ \\
\hline Diastolic blood pressure (mmHg) & $77.4(12.2)$ & $77.1(11.3)$ & $77.0(11.2)$ & $78.8(13.7)$ & $\mathrm{NS}^{*}$ \\
\hline
\end{tabular}

* Significant differences tested with linear independent pairwise comparison adjusting for age and gender. NS - not significant $(P>0.05)$.

† Significant difference tested with $t$-test, adjusted for age, area of residence and year of study.

study, no significant difference in anthropometric variables and blood pressures was observed.

Table 3 lists all of the metabolic (biochemical) variables that differed significantly, as well as those with no significant differences, between HIV-infected and uninfected subjects when data were adjusted for age, gender, area of residence and year of study. Haemoglobin levels of the infected subjects were significantly lower, without differences in the other iron status variables (serum iron, ferritin, transferrin and iron-binding capacity). Total serum protein and globulin were significantly higher while albumin was significantly lower in infected subjects. Plasma fibrinogen, a major acute-phase blood protein, did not differ significantly. Total serum cholesterol,

Table 3 Variables that differed significantly and did not differ between HIV-infected and uninfected subjects

\begin{tabular}{|c|c|c|c|c|c|c|c|}
\hline \multirow[b]{2}{*}{ Variable } & \multicolumn{3}{|c|}{$\begin{array}{l}\text { HIV-infected } \\
\quad(n=212)\end{array}$} & \multicolumn{3}{|c|}{$\begin{array}{l}\text { HIV-uninfected } \\
\quad(n=1523)\end{array}$} & \multirow[b]{2}{*}{$P$-value } \\
\hline & Mean* & SE & $95 \% \mathrm{Cl}$ & Mean* & SE & $95 \% \mathrm{Cl}$ & \\
\hline \multicolumn{8}{|l|}{$\begin{array}{l}\text { Variables that differed } \\
\text { Blood }\end{array}$} \\
\hline Haematocrit (\%) & 41.4 & 0.32 & $40.7-41.9$ & 43.1 & 0.12 & $42.9-43.3$ & 0.000 \\
\hline Haemoglobin $\left(\mathrm{gl}^{-1}\right)$ & 12.4 & 0.14 & $12.2-12.7$ & 12.8 & 0.05 & $12.7-12.9$ & 0.029 \\
\hline \multicolumn{8}{|l|}{ Serum } \\
\hline Albumin $\left(\mathrm{gl}^{-1}\right)$ & 41.3 & 0.30 & $40.6-41.7$ & 43.6 & 0.11 & $43.4-43.8$ & 0.000 \\
\hline Globulin $\left(\mathrm{gl}^{-1}\right)$ & 36.8 & 0.43 & $36.0-37.7$ & 30.3 & 0.16 & $30.0-30.6$ & 0.000 \\
\hline Total protein $\left(\mathrm{gl}^{-1}\right)$ & 78.1 & 0.46 & $77.0-78.8$ & 73.8 & 0.17 & $73.5-74.1$ & 0.000 \\
\hline Total cholesterol $\left(\mathrm{mmol}^{-1}\right)$ & 3.90 & 0.07 & $3.78-4.05$ & 4.17 & 0.03 & $4.12-4.22$ & 0.000 \\
\hline HDL-cholesterol $\left(\mathrm{mmoll}^{-1}\right)$ & 1.09 & 0.02 & $1.04-1.13$ & 1.19 & 0.01 & $1.17-1.21$ & 0.000 \\
\hline Triglycerides $\left(\mathrm{mmoll}^{-1}\right)$ & 1.09 & 0.05 & $0.99-1.19$ & 1.21 & 0.02 & $1.17-1.24$ & 0.039 \\
\hline $\operatorname{ALT}\left(\mathrm{IUI}^{-1}\right)$ & 15.6 & 1.0 & $14.0-17.7$ & 12.2 & 0.4 & $11.5-12.9$ & 0.000 \\
\hline AST $\left(\mathrm{IUI}^{-1}\right)$ & 31.7 & 2.4 & $27.1-36.3$ & 22.1 & 0.9 & $20.4-23.8$ & 0.000 \\
\hline LD $\left(I U^{-1}\right)^{\prime}$ & 140.4 & 2.3 & $135.9-144.9$ & 132.5 & 0.9 & $130.8-134.2$ & 0.001 \\
\hline \multicolumn{8}{|l|}{$\begin{array}{l}\text { Variables that did not differ } \\
\text { Serum }\end{array}$} \\
\hline Sodium $\left(\mathrm{mmoll}^{-1}\right)$ & 137.2 & 0.35 & $136.6-137.9$ & 137.8 & 0.13 & $137.5-138.0$ & 0.134 \\
\hline Potassium (mmoll $\left.{ }^{-1}\right)$ & 3.9 & 0.03 & $3.9-4.1$ & 3.9 & 0.01 & $3.9-4.0$ & 0.973 \\
\hline Chloride (mmoll $\left.{ }^{-1}\right)$ & 103.3 & 0.24 & $102.8-103.8$ & 103.8 & 0.09 & $103.6-103.9$ & 0.061 \\
\hline Urea $\left(\mathrm{mmoll}^{-1}\right)$ & 3.4 & 0.09 & $3.3-3.6$ & 3.6 & 0.03 & $3.5-3.6$ & 0.099 \\
\hline Uric acid $\left(\mathrm{mmoll}^{-1}\right)$ & 0.3 & 0.009 & $0.29-0.33$ & 0.3 & 0.003 & $0.29-0.31$ & 0.253 \\
\hline Creatinine $\left(\mathrm{mmoll}^{-1}\right)$ & 83.5 & 0.94 & $81.6-85.3$ & 82.4 & 0.35 & $81.7-83.1$ & 0.299 \\
\hline Calcium (mmoll-1) & 2.3 & 0.006 & $2.3-2.3$ & 2.3 & 0.002 & $2.3-2.3$ & 0.537 \\
\hline Magnesium (mmoll $\left.{ }^{-1}\right)$ & 0.83 & 0.006 & $0.82-0.84$ & 0.84 & 0.002 & $0.83-0.84$ & 0.119 \\
\hline Iron $\left(\mathrm{mmoll}^{-1}\right)$ & 16.4 & 0.59 & $15.2-17.6$ & 16.9 & 0.222 & $16.5-17.4$ & 0.407 \\
\hline TIBC (mmoll $\left.{ }^{-1}\right)$ & 66.2 & 0.92 & $64.4-68.0$ & 67.5 & 0.341 & $66.8-68.2$ & 0.200 \\
\hline$\%$ Iron saturation & 25.9 & 0.98 & $24.1-27.9$ & 25.9 & 0.36 & $25.2-26.6$ & 0.965 \\
\hline Ferritin $\left(\mathrm{mg} \mathrm{l}^{-1}\right)$ & 133.4 & 17.5 & $99-167.8$ & 141.5 & 6.5 & $128.7-154.3$ & 0.665 \\
\hline Fibrinogen $\left(\mathrm{gl}^{-1}\right)$ & 3.4 & 0.08 & $3.3-3.6$ & 3.4 & 0.3 & $3.4-3.5$ & 0.852 \\
\hline Glucose $\left(\mathrm{mmoll}^{-1}\right)$ & 4.7 & 0.115 & $4.5-4.9$ & 4.8 & 0.042 & $4.6-4.9$ & 0.408 \\
\hline Vitamin $A\left(\mu \mathrm{g} \mathrm{dl}^{-1}\right)$ & 44.4 & 1.29 & $41.9-46.9$ & 46.7 & 0.47 & $45.8-47.6$ & 0.104 \\
\hline Vitamin $E\left(\mathrm{mgl}^{-1}\right)$ & 8.7 & 0.25 & $8.17-9.15$ & 9.03 & 0.9 & $8.8-9.2$ & 0.169 \\
\hline
\end{tabular}

SE - standard error; Cl - confidence interval; HDL - high-density lipoprotein; ALT - alanine aminotransferase; AST - aspartate aminotransferase; LD - lactate dehydrogenase; TIBC - total iron-binding capacity.

* Estimated marginal mean.

† Linear independent pairwise comparison adjusted for age, area of residence, year of study and gender. 
high-density lipoprotein (HDL)-cholesterol and triglycerides were significantly lower in the infected subjects. Three of the measured liver enzymes in serum, alanine aminotransferase (ALT), aspartate aminotransferase (AST) and lactate dehydrogenase (LD), were statistically significantly higher in the infected subjects, reflecting tissue and cell damage in these asymptomatic subjects. None of the many other biochemical variables measured, including several serum/plasma vitamin levels, differed significantly between the two groups when controlled for age, gender, year of the study and area of residence.

Table 4 presents mean nutrient intake data according to HIV status and gender. There was no statistically significant difference in mean nutrient intakes between HIV-infected and uninfected subjects. Adjusting for age, gender, area of residence and year of study made little difference to the relationships seen for unadjusted male and female data. Compared with dietary guidelines ${ }^{23}$, it seems that all groups followed a 'prudent' diet with total fat providing less than $26 \%$ of total energy. Based on US recommended dietary allowances ${ }^{22}$ folate intake appeared to be adequate, but mean intakes of most other micronutrients were less than the recommended dietary allowances. Although macronutrient intakes were within the range considered desirable and prudent to reduce risk of non-communicable chronic diseases, intake of micronutrients was not adequate.

Table 5 shows significant partial correlations (controlled for age, gender, year of study and area of residence) of haemoglobin, serum albumin, AST and total cholesterol. These variables were selected because of the significant differences in mean values of infected and uninfected subjects, and because the patterns of correlations in the serum lipids were similar, as were those in the liver enzymes. In the HIV-infected subjects, correlations were generally higher; many of those observed in uninfected subjects disappeared while a few new ones emerged.

Serum albumin in HIV-infected subjects showed a high negative correlation with serum globulin $(r=-0.62$, $P=0.001)$ and positive correlations with serum cholesterol, HDL-cholesterol, haemoglobin, vitamins A and E, as well as serum iron and total iron-binding capacity. Serum albumin did not correlate with the liver enzymes in these subjects. The latter correlated positively with each other and with serum glucose. These liver enzymes, indicative of cell damage and probably an inflammatory response, all correlated positively with total dietary fat intake, and specifically with polyunsaturated fat intake (correlations of $r=+0.21,+0.22,+0.41$ and $+0.63, P \leq 0.005$ with alkaline phosphatase, $\gamma$-glutamyl transferase, ALT and AST, respectively).

A principal components analysis was used to summarise dietary patterns. The first principal component (which explained $13 \%$ of the variation) highlighted the contrast in intakes between meat, fruit and vegetables in one direction and the consumption of mainly staples (maize meal): a high positive score reflected a diet higher in meat and vegetable products, and a more negative score reflected a diet higher in maize meal porridge and samp. Table 6 presents the mean dietary score derived from this first principal component for each third of the distribution of AST for HIV-infected subjects only. This shows that

Table 4 Comparison of habitual mean (standard deviation) dietary intakes of HIV-infected and uninfected men and women

\begin{tabular}{|c|c|c|c|c|c|}
\hline \multirow[b]{2}{*}{ Variable } & \multicolumn{2}{|c|}{ Men } & \multicolumn{2}{|c|}{ Women } & \multirow[b]{2}{*}{$\begin{array}{c}\mathrm{RDA}^{23} \text { and dietary } \\
\text { guidelines }\end{array}$} \\
\hline & $\begin{array}{l}\text { HIV-infected } \\
(n=93)\end{array}$ & $\begin{array}{l}\text { HIV-uninfected } \\
\quad(n=654)\end{array}$ & $\begin{array}{l}\text { HIV-infected } \\
(n=119)\end{array}$ & $\begin{array}{l}\text { HIV-uninfected } \\
\quad(n=896)\end{array}$ & \\
\hline Energy (kJ) & $10099(4475)$ & $9487(3787)$ & $8271(3136)$ & 7933 (3053) & \\
\hline Plant protein (g) & $39.6(19.8)$ & $36.4(16.8)$ & $32.5(14.5)$ & $30.6(13.7)$ & \\
\hline Animal protein (g) & $28.4(16.2)$ & $29.6(16.6)$ & $26.2(14.8)$ & $26.9(16.1)$ & \\
\hline Total fat (g) & $60.7(28.8)$ & $58.9(26.7)$ & $56.6(27.9)$ & $54.1(25.6)$ & \\
\hline $\mathrm{P} / \mathrm{S}$ ratio & $0.98(0.50)$ & $0.89(0.36)$ & $0.88(0.38)$ & $0.88(0.37)$ & 1.0 \\
\hline Cholesterol (mg) & $345(258)$ & $343(225)$ & $279(191)$ & $284(204)$ & \\
\hline Carbohydrate (g) & $361(162)$ & 341 (153) & $311(131)$ & $293(131)$ & \\
\hline Added sugar (g) & $54.2(41.8)$ & $54.0(47.4)$ & 55.7 (47.9) & 51.7 (47.6) & \\
\hline Dietary fibre $(\mathrm{g})$ & $19.6(10.5)$ & $18.1(9.1)$ & $17.2(8.2)$ & $16.2(7.5)$ & $25-30$ \\
\hline Protein (\% of energy) & $11.8(2.1)$ & $12.0(2.1)$ & $11.6(2.1)$ & $11.9(2.2)$ & $10-15$ \\
\hline Fat ( $\%$ of energy) & $24.4(7.2)$ & $24.9(7.6)$ & $25.5(7.0)$ & $25.9(7.5)$ & $<30$ \\
\hline Carbohydrate (\% of energy) & $65.8(9.3)$ & $64.8(9.9)$ & $64.5(9.4)$ & $63.8(10.0)$ & $>55$ \\
\hline Calcium (mg) & $448(248)$ & $462(271)$ & $405(213)$ & $408(246)$ & $800-1200$ \\
\hline Magnesium (mg) & 369 (212) & 354 (178) & 304 (129) & $287(126)$ & $320-420$ \\
\hline Phosphorus (mg) & 1214 (592) & 1163 (484) & $991(367)$ & $955(375)$ & 700 \\
\hline Potassium (mg) & $2282(902)$ & $2260(905)$ & $2124(853)$ & 2020 (793) & - \\
\hline Sodium (mg) & $1457(980)$ & $1348(778)$ & $1271(701)$ & 1232 (705) & - \\
\hline Zinc (mg) & $9.1(4.3)$ & $8.9(3.8)$ & $8.2(3.4)$ & $8.0(3.5)$ & $8-11$ \\
\hline Iron (mg) & $9.1(4.5)$ & $9.2(4.4)$ & $8.9(4.3)$ & $8.5(4.1)$ & $10-15$ \\
\hline Vitamin A (RE) & $763(651)$ & $696(619)$ & $741(577)$ & 764 (731) & $800-1000$ \\
\hline Ascorbic acid (mg) & $33.4(31.8)$ & 349 (35.5) & $40.0(46.7)$ & $38.8(42.0)$ & 60 \\
\hline Folate $(\mu \mathrm{g})$ & $237(136)$ & 221 (98.9) & $197(81)$ & $182(82)$ & $150-200$ \\
\hline
\end{tabular}

RDA - US recommend dietary allowances in ranges for adult men and women; P/S ratio - ratio of polyunsaturated fat to saturated fat; RE - retinol equivalents. 
Table 5 Significant partial correlations* of selected variables that differed significantly between HIV-infected and uninfected subjects

\begin{tabular}{|c|c|c|c|c|c|}
\hline \multicolumn{3}{|c|}{ HIV-infected } & \multicolumn{3}{|c|}{ HIV-uninfected } \\
\hline Variable & $r$ & $P$-value & Variable & $r$ & $P$-value \\
\hline $\begin{array}{l}\text { Haemoglobin } \\
\mathrm{S} \mathrm{Na} \\
\mathrm{S} \mathrm{CO}_{2} \\
\mathrm{~S} \mathrm{Ca} \\
\mathrm{S} \text { albumin } \\
\mathrm{S} \text { globulin } \\
\mathrm{S} \mathrm{TC} \\
\mathrm{S} \text { vit A } \\
\mathrm{S} \text { vit } \mathrm{E} \\
\mathrm{S} \mathrm{Fe} \\
\text { Dietary fibre } \\
\text { Dietary thiamin } \\
\text { Dietary folate } \\
\text { Alcohol intake }\end{array}$ & $\begin{array}{l}+0.17 \\
+0.23 \\
+0.18 \\
+0.25 \\
-0.17 \\
+0.17 \\
+0.19 \\
+0.26 \\
+0.19 \\
-0.16 \\
-0.14 \\
-0.14 \\
+0.13\end{array}$ & $\begin{array}{l}0.029 \\
0.003 \\
0.019 \\
0.001 \\
0.028 \\
0.027 \\
0.017 \\
0.001 \\
0.014 \\
0.020 \\
0.040 \\
0.040 \\
0.06\end{array}$ & $\begin{array}{l}\text { Haemoglobin } \\
\mathrm{S} \mathrm{Na} \\
\mathrm{S} \mathrm{K} \\
\mathrm{S} \mathrm{CO}_{2} \\
\mathrm{~S} \mathrm{Ca} \\
\mathrm{S} \mathrm{Mg} \\
\mathrm{S} \text { albumin } \\
\mathrm{S} \mathrm{T} \text { bilirubin } \\
\mathrm{S} \mathrm{TC} \\
\mathrm{S} \mathrm{LDL}-\mathrm{C} \\
\mathrm{S} \text { vit A } \\
\mathrm{S} \text { vit E } \\
\mathrm{S} \mathrm{Fe} \\
\mathrm{S} \text { Fe sat } \\
\text { Plasma fibrinogen } \\
\text { Alcohol intake }\end{array}$ & $\begin{array}{l}+0.06 \\
+0.11 \\
+0.16 \\
+0.22 \\
+0.08 \\
+0.24 \\
+0.14 \\
+0.08 \\
+0.06 \\
+0.12 \\
+0.06 \\
+0.22 \\
+0.20 \\
+0.06 \\
+0.06\end{array}$ & $\begin{array}{l}0.001 \\
0.001 \\
0.001 \\
0.006 \\
0.001 \\
0.001 \\
0.001 \\
0.008 \\
0.028 \\
0.001 \\
0.034 \\
0.001 \\
0.001 \\
0.034 \\
0.032\end{array}$ \\
\hline $\begin{array}{l}\text { Serum albumin } \\
\text { S Na } \\
\text { S K } \\
\text { S Cl } \\
\text { S Ca } \\
\text { S Mg } \\
\text { S prot } \\
\text { S globulin } \\
\text { S T bilirubin } \\
\text { S TC } \\
\text { S HDL-C } \\
\text { S LDL-C } \\
\text { Hc } \\
\text { Hb } \\
\text { S vit A } \\
\text { S vit E } \\
\text { S Fe } \\
\text { S TIBC }\end{array}$ & $\begin{array}{l}+0.50 \\
+0.17 \\
+0.23 \\
+0.77 \\
+0.36 \\
-0.20 \\
-0.62 \\
+0.20 \\
+0.52 \\
+0.41 \\
+0.31 \\
+0.27 \\
+0.25 \\
+0.40 \\
+0.29 \\
+0.27 \\
+0.38\end{array}$ & $\begin{array}{l}0.001 \\
0.035 \\
0.004 \\
0.001 \\
0.001 \\
0.009 \\
0.001 \\
0.01 \\
0.001 \\
0.001 \\
0.001 \\
0.001 \\
0.001 \\
0.001 \\
0.001 \\
0.001 \\
0.001\end{array}$ & $\begin{array}{l}\text { Serum albumin } \\
\text { S Na } \\
\text { S K } \\
\text { S Cl } \\
\text { S CO } 2 \\
\text { S urea } \\
\text { S uric acid } \\
\text { S creat } \\
\text { S Ca } \\
\text { S Mg } \\
\text { S prot } \\
\text { S T bilirubin } \\
\text { S ALT } \\
\text { S AST } \\
\text { S LD } \\
\text { S TC } \\
\text { S HDL-C } \\
\text { S LDL-C } \\
\text { S TG } \\
\text { HC } \\
\text { Hb } \\
\text { S vit A } \\
\text { S vit E } \\
\text { S Fe } \\
\text { S TIBC } \\
\text { S Fe sat } \\
\text { Plasma fibrinogen }\end{array}$ & $\begin{array}{l}+0.24 \\
+0.16 \\
+0.19 \\
+0.15 \\
+0.07 \\
+0.07 \\
+0.10 \\
+0.64 \\
+0.37 \\
+0.59 \\
+0.13 \\
+0.09 \\
+0.07 \\
+0.16 \\
+0.28 \\
+0.13 \\
+0.21 \\
+0.08 \\
+0.26 \\
+0.24 \\
+0.31 \\
+0.17 \\
+0.18 \\
+0.16 \\
+0.11 \\
-0.14\end{array}$ & $\begin{array}{l}0.001 \\
0.001 \\
0.001 \\
0.001 \\
0.009 \\
0.019 \\
0.001 \\
0.001 \\
0.001 \\
0.001 \\
0.001 \\
0.002 \\
0.024 \\
0.001 \\
0.001 \\
0.001 \\
0.001 \\
0.008 \\
0.001 \\
0.001 \\
0.001 \\
0.001 \\
0.001 \\
0.001 \\
0.001 \\
0.001\end{array}$ \\
\hline $\begin{array}{l}\text { AST } \\
\text { S Na } \\
\text { S Cl } \\
\text { S uric acid } \\
\text { S Fe } \\
\text { S T bilirubin } \\
\text { S D bilirubin } \\
\text { S ALP } \\
\text { S GGT } \\
\text { S ALT } \\
\text { S gluc } \\
\text { S Fe sat } \\
\text { Dietary total fat } \\
\text { Dietary PUFA }\end{array}$ & $\begin{array}{l}-0.15 \\
-0.20 \\
+0.18 \\
-0.15 \\
+0.39 \\
+0.43 \\
+0.30 \\
+0.41 \\
+0.74 \\
+0.27 \\
+0.38 \\
+0.18 \\
+0.41\end{array}$ & $\begin{array}{l}0.05 \\
0.009 \\
0.025 \\
0.05 \\
0.001 \\
0.001 \\
0.001 \\
0.001 \\
0.001 \\
0.001 \\
0.001 \\
0.014 \\
0.001\end{array}$ & $\begin{array}{l}\text { AST } \\
\mathrm{S} \mathrm{CO}_{2} \\
\text { S urea } \\
\text { S uric acid } \\
\text { S prot } \\
\text { S albumin } \\
\text { S globulin } \\
\text { S T bilirubin } \\
\text { S D bilirubin } \\
\text { S GGT } \\
\text { S ALT } \\
\text { S LD } \\
\text { S TC } \\
\text { S HDL-C } \\
\text { S TG } \\
\text { S vit A } \\
\text { S Fe } \\
\text { S Fe sat } \\
\text { S ferritin }\end{array}$ & $\begin{array}{l}-0.10 \\
-0.10 \\
+0.07 \\
+0.17 \\
+0.07 \\
+0.15 \\
+0.07 \\
+0.08 \\
+0.39 \\
+0.58 \\
+0.22 \\
+0.13 \\
+0.19 \\
+0.21 \\
+0.14 \\
+0.19 \\
+0.18 \\
+0.29\end{array}$ & $\begin{array}{l}0.001 \\
0.001 \\
0.02 \\
0.001 \\
0.024 \\
0.001 \\
0.013 \\
0.005 \\
0.001 \\
0.001 \\
0.001 \\
0.001 \\
0.001 \\
0.001 \\
0.001 \\
0.001 \\
0.001 \\
0.001\end{array}$ \\
\hline $\begin{array}{l}\text { Total cholesterol } \\
\text { S Na } \\
\mathrm{S} \mathrm{Ca} \\
\mathrm{S} \mathrm{Mg}\end{array}$ & $\begin{array}{l}+0.27 \\
+0.54 \\
+0.30\end{array}$ & $\begin{array}{l}0.001 \\
0.001 \\
0.001\end{array}$ & $\begin{array}{l}\text { Total cholesterol } \\
\mathrm{S} \mathrm{Na} \\
\mathrm{S} \mathrm{K} \\
\mathrm{S} \mathrm{CO}_{2}\end{array}$ & $\begin{array}{l}+0.11 \\
+0.09 \\
+0.09\end{array}$ & 0.001 \\
\hline
\end{tabular}


Table 5 Continued

\begin{tabular}{|c|c|c|c|c|c|}
\hline \multicolumn{3}{|c|}{ HIV-infected } & \multicolumn{3}{|c|}{ HIV-uninfected } \\
\hline Variable & $r$ & $P$-value & Variable & $r$ & $P$-value \\
\hline $\begin{array}{l}\text { S albumin } \\
\text { S globulin } \\
\text { S LD } \\
\text { S HDL-C } \\
\text { S LDL-C } \\
\text { S TG } \\
\text { Hb } \\
\text { S vit A } \\
\text { S vit E } \\
\text { S TIBC } \\
\text { Dietary saturated fat } \\
\text { S Ca }\end{array}$ & $\begin{array}{l}+0.52 \\
-0.26 \\
+0.18 \\
+0.32 \\
+0.88 \\
+0.27 \\
+0.17 \\
+0.25 \\
+0.43 \\
+0.24 \\
+0.14 \\
+0.15\end{array}$ & $\begin{array}{l}0.001 \\
0.001 \\
0.019 \\
0.001 \\
0.001 \\
0.001 \\
0.027 \\
0.001 \\
0.001 \\
0.001 \\
0.07 \\
0.05\end{array}$ & $\begin{array}{l}\text { S urea } \\
\text { S uric acid } \\
\text { S creat } \\
\text { S Fe } \\
\text { S Ca } \\
\text { S Mg } \\
\text { S prot } \\
\text { S albumin } \\
\text { S globulin } \\
\text { S GGT } \\
\text { S ALT } \\
\text { S AST } \\
\text { S LD } \\
\text { S HDL-C } \\
\text { S LDL-C } \\
\text { S TG } \\
\text { Hc } \\
\text { Hb } \\
\text { S gluc } \\
\text { S vit A } \\
\text { S vit E } \\
\text { S TIBC } \\
\text { Energy } \\
\text { Plant protein } \\
\text { Thiamin }\end{array}$ & $\begin{array}{l}+0.11 \\
+0.12 \\
+0.12 \\
+0.07 \\
+0.29 \\
+0.14 \\
+0.28 \\
+0.27 \\
+0.98 \\
+0.67 \\
+0.11 \\
+0.12 \\
+0.13 \\
+0.22 \\
+0.91 \\
+0.33 \\
+0.11 \\
+0.078 \\
+0.06 \\
+0.24 \\
+0.53 \\
+0.12 \\
-0.06 \\
-0.08 \\
-0.055\end{array}$ & $\begin{array}{l}0.001 \\
0.001 \\
0.001 \\
\\
0.001 \\
0.001 \\
0.001 \\
0.001 \\
0.001 \\
\\
0.001 \\
0.001 \\
0.001 \\
0.001 \\
0.001 \\
0.001 \\
0.001 \\
0.088 \\
0.042 \\
0.001 \\
0.001 \\
0.001 \\
0.025 \\
0.002 \\
0.043\end{array}$ \\
\hline
\end{tabular}

S - serum; TC - total cholesterol; vit A - vitamin A; vit E - vitamin E; prot - protein; T bilirubin - total bilirubin; D bilirubin - direct (conjugated) bilirubin; HDL-C - high-density lipoprotein cholesterol; LDL-C - low-density lipoprotein cholesterol; Hc - haematocrit; $\mathrm{Hb}$ - haemoglobin; TIBC - total iron-binding capacity; ALP - alkaline phosphatase; GGT - $\gamma$-glutamyl transferase, gluc - glucose; Fe sat - \% iron saturation; PUFA - polyunsaturated fatty acids; LD - lactate dehydrogenase; TG triglycerides; creat - creatinine; ALT - alanine aminotransferase; AST - aspartate aminotransferase.

* Controlled for age, gender, year of study and area of residence (urban, rural).

those subjects in the lowest third of AST had a statistically significantly higher mean dietary score than subjects in the middle and highest third of AST. There were also statistically significant negative Spearman correlations between each of the three liver enzymes (AST, LD, ALT) and the diet score, suggesting that subjects with the highest liver enzyme levels were more likely to have a diet lower in meat and vegetables.

The dietary score was included in a regression model together with other variables (such as gender, age, area of residence) and HIV status, with albumin as the outcome measure. Albumin was chosen as a marker of deterioration in the metabolic profile. When controlling for all other variables in the model, albumin rose by $2.52 \mathrm{gl}^{-1}$ from HIV-uninfected to infected subjects; the effect of the first principal component was statistically significant, even after adjusting for the effects of all other variables in the model.

Table 7 shows the odds ratios and 95\% confidence intervals of albumin, globulin, AST and total cholesterol in thirds for HIV-infected compared with HIV-uninfected subjects, with the lowest third as reference category.

\section{Discussion}

The THUSA study was not designed to measure the prevalence or incidence of HIV infection. The main objective was to assess the impact of urbanisation on health risks in 'apparently healthy', disease-free volunteers. The data on these 216 asymptomatic HIV-infected subjects are nevertheless important because the nutritional status and biochemical nutritional profile of these subjects, compared with the rest of the HIV-uninfected study population, may provide useful information for examining appropriate, evidence-based dietary guidelines for HIVinfected Africans.

The absence of significant differences between HIVinfected and uninfected subjects in body mass index (BMI) and total sum of skinfold thicknesses probably indicates that the majority of the HIV-infected but asymptomatic subjects were at an early stage of the infection. Weight loss and wasting of HIV/AIDS result from diarrhoea and malabsorption, increased nutrient requirements due to infections, altered metabolism and decreased intakes ${ }^{12}$. Kotler $^{20}$ mentions that a decreased body cell mass is observed early in HIV infection. It predates significant immune suppression, indicating that the virus itself may be involved. Body cell mass was not determined in this study, but total fat percentage calculated from the seven skinfold thicknesses did not differ significantly between infected and uninfected subjects. Furthermore, there were virtually no differences in mean nutrient intakes of HIV-infected and uninfected subjects. The biochemical variables 
Table 6 Mean diet score* and 95\% confidence interval $(\mathrm{Cl})$ by thirds of liver enzyme (aspartate aminotransferase, AST) in HIVinfected subjects

\begin{tabular}{lrrr}
\hline Thirds of AST & Mean & $95 \% \mathrm{Cl}$ & \multicolumn{1}{c}{$\begin{array}{c}\text { Overall } \\
F \text {-ratio; } P \text {-value }\end{array}$} \\
\hline Lowest & 0.17 & $0.08,0.43$ & $3.04 ; P=0.05$ \\
Middle & -0.19 & $-0.41,0.03$ & \\
Highest & -0.16 & $-0.37,0.06$ & \\
\hline
\end{tabular}

Spearman correlations, liver enzymes and diet score: alanine aminotransferase, $-0.19(P=0.007)$; AST, $-0.15(P=0.031)$.

* Score derived from first principal component: positive score indicates higher consumption of meat products and vegetables; negative score indicates higher consumption of maize meal and lower consumption of meat and vegetables. Adjusted for age, area of residence, year of study and gender.

Table 7 Logistic regression analysis (odds ratios (95\% confidence interval)) of biochemical measures in thirds for HIV-infected compared with HIV-uninfected subjects

\begin{tabular}{lcc}
\hline Variable & Middle third & Upper third \\
\hline Albumin & $0.58(0.40-0.85)$ & $0.37(0.24-0.57)$ \\
Globulin & $1.46(0.92-2.33)$ & $3.93(2.55-6.04)$ \\
Aspartate aminotransferase & $1.47(0.95-2.28)$ & $2.13(1.49-3.66)$ \\
Total cholesterol & $0.67(0.46-0.98)$ & $0.58(0.37-0.91)$ \\
\hline
\end{tabular}

Adjusted for age, gender, area of residence and year of study, in thirds of each measure (lowest third as reference category).

indicative of nutritional status that differed significantly between the two groups (Table 3) could therefore reflect early effects of the virus and the body's responses to these effects. The two acute-phase proteins measured, plasma fibrinogen and serum ferritin, did not differ significantly between groups, possibly indicating that the acute-phase response was not operative in the HIV subjects. However, early tissue damage and an inflammatory response were probably present, revealed by the statistically significantly higher ALT, AST and LD values, as well as the decreased albumin levels of the infected persons. The significantly lower haematocrit and haemoglobin levels of the HIVinfected subjects were not accompanied by differences in serum iron, ferritin, transferrin or total iron-binding capacity. The early anaemia reported for HIV-infected subjects seems therefore not to be dietary related. The significant correlations between haemoglobin and albu$\min (r=+0.25, \quad P=0.001), \quad$ globulin $(r=-0.17$, $P=0.028)$, serum vitamin A $(r=+0.19, \quad P=0.017)$, vitamin $\mathrm{E}(r=+0.26, P=0.001)$ and iron $(r=+0.19$, $P=0.014$ ) suggest, however, that maintaining an 'optimal' nutrition status and preventing a decrease in albumin may be associated with higher levels of haemoglobin. The mechanism remains obscure. It is intriguing that consumption of dietary fibre showed a significant negative correlation with haemoglobin in the infected subjects (the other two correlates, thiamin and folate, may be related to dietary fibre since they share similar food sources). This suggests that consumption of foods rich in fibre may be associated with lower haemoglobin values in HIV-infected persons. The absence of decreases in serum iron and other iron status variables argues against an inhibition of iron absorption. At a later stage of the disease ${ }^{10,24}$ in African patients who had BMI of $18.9 \mathrm{~kg} \mathrm{~m}^{-2}$ (men) and $22.7 \mathrm{~kg} \mathrm{~m}^{-2}$ (women) $)^{10}$, the decreased haematocrit and haemoglobin values were accompanied by decreased serum vitamin $\mathrm{B}_{12}$ and erythrocyte folate values ${ }^{24}$, indicating a diet-induced anaemia.

Decreased serum albumin and increased globulin and total protein levels have been found in HIV-infected subjects and AIDS patients by several authors (reviewed by Gramlich and Mascioli ${ }^{13}$ ). Although the HIV-infected subjects in this study still had high albumin levels, their mean level was significantly lower than that of noninfected subjects, reflecting a possible decrease in nutrition status, even at this early stage. Van Staden et $a l^{24}$ reported a positive correlation between serum albumin and total serum cholesterol. This study supports that observation. The relationship between serum albumin and total cholesterol of $r=+0.28$ $(P=0.001)$ in the HIV-uninfected subjects increased to $r=+0.52(P=0.001)$ in the infected subjects (Table 5). As in the case for haemoglobin, the significant positive correlations of albumin with serum vitamins $\mathrm{A}$ and $\mathrm{E}$, serum iron and total iron-binding capacity suggest that albumin levels will be higher in subjects with good nutrition status.

The decreased total serum cholesterol observed in our HIV-infected subjects was also reported by others ${ }^{25,26}$. As for serum albumin ${ }^{12}$, there appears to be an association between disease stage and serum cholesterol level ${ }^{25}$. The decrease in cholesterol of the HIV-infected subjects was accompanied by a decrease in HDL-cholesterol. Serum glucose and insulin levels during the glucose tolerance tests did not differ significantly between infected and uninfected subjects. It therefore appears that the early alterations in serum albumin and lipids are not insulinrelated. The reason for and mechanism of the observed decreases in serum lipids at this early stage of HIV infection, even before weight or other anthropometric changes are present, are not clear. The correlates of total serum cholesterol (Table 5) suggest that those infected subjects with higher levels of albumin, serum vitamins A and $\mathrm{E}$ and serum iron (therefore, with a 'better' nutrition status) had less reductions in serum cholesterol. In the infected subjects, the significant correlation between consumption of saturated fat (from animal products) and serum total cholesterol further suggests that in this instance, a diet rich in animal products may be beneficial in preventing this decrease.

The significant positive correlations between the consumption of polyunsaturated fat and liver enzymes (Table 5) may be an indication that these fatty acids (mainly sunflower oil in the diets of the participating subjects) should be limited in the diet of HIV-infected persons. Although these subjects were on a relatively 
low-fat (prudent) diet (see Table 3), the results suggest that, even at this low level of fat intake, polyunsaturates are associated with increased liver cell damage.

The question arises of whether the observed deterioration in haemoglobin, albumin and serum lipids can be prevented by a better diet. The THUSA subjects followed a prudent, low-fat, high-carbohydrate diet, which was micronutrient-deficient. The principal components analysis suggests that HIV-infected subjects who followed this dietary pattern were more likely to have higher liver enzyme levels, suggestive of early metabolic changes. Those HIV-infected subjects who consumed a diet higher in meat and vegetables were likely to have what might be considered a 'better' metabolic state as indicated with higher levels of the mentioned nutritional status variables. It is not clear which nutrients might be responsible for these associations. The intake of a number of nutrients would be expected to differ between those on a diet higher in meat (animal protein, saturated fat, riboflavin, calcium, phosphorus, zinc), fresh fruits and vegetables (ascorbic acid, magnesium, potassium) compared with a diet based mainly on maize meal. In summary, Table 7 suggests that among those who are HIV-positive, those with a diet score highlighting higher consumption of meat and vegetables, in contrast with more maize-based diets, had lower liver enzyme levels.

Is there a dietary pattern that might be optimal to reduce the rate of progression of HIV/AIDS? The results of this study suggest that there may be. The results indicated that an 'affluent, Western' type of diet, different from the prudent diet recommended for optimal health ${ }^{21,27}$, is positively associated with metabolic variables known to decrease in HIV-infected subjects proportionally to disease stage ${ }^{12,25}$. Urgent research is necessary for a better understanding of the early metabolic effects of HIV and ways that diet can be used to reduce the impact of HIV infection on health. This study gives an indication that there is a need to acknowledge that what might be considered a 'healthy' diet in the general population with a low HIV/AIDS prevalence might not be optimal for a population with a high incidence of HIV/AIDS. Should the results of this study be confirmed by future research, it could have a profound influence on the development of dietary guidelines and possibly of food supplements for populations with high HIV/AIDS prevalence. It is suggested that the possible 'protective' effect of a diet containing sufficient amounts of meat, fruit and vegetables, rather than of mainly maize-based staple foods, should be examined in HIV-infected subjects.

\section{Acknowledgements}

The THUSA study was funded by grants from the National Research Foundation, South African Medical Research Council, South African Sugar Association, The Dry Bean Producers Organization and Potchefstroom University.

\section{References}

1 Joint United Nations Programme on HIV/AIDS (UNAIDS). AIDS Epidemic Update: December 1998. Geneva: World Health Organization, 1998.

2 Foster G. Today's children - challenges to child health promotion in countries with severe AIDS epidemics. AIDS Care 1998; 10(1): S17-S23.

3 Fauci AS. The AIDS epidemic. Considerations for the 21st century. New England Journal of Medicine 1999; 341(14): $1046-50$.

4 Anon. Health and related indicators. In: Ntuli A, ed. South African Health Review 1998. Durban: Health Systems Trust, 1998; 203-15.

5 Day C, Gray A. Health and related indicators. In: Ijumba P, Ntuli A, Barron P, eds. South African Health Review 2002. Durban: Health Systems Trust, 2002; 411-533.

6 North West Province Department of Health. Results of the HIV sero-prevalence survey of women attending antenatal clinics in the North West Province, 1998. Mmabatho: Department of Health, North West Province of South Africa, 1999; 1-11.

7 South African Government Department of Health. HIV and AIDS Care, Management and Treatment for South Africa [online], 2003. Available at: http://www.gov.za/reports/ 2003/aidsplan/report.pdf. Accessed 8 March 2004.

8 South African Government Department of Health. South African National Guidelines on Nutrition for People living with TB, HIV/AIDS and Other Chronic Debilitating Conditions. Pretoria: Department of Health, South Africa, 2001; $1-28$.

9 Morgan D, Maude GH, Malamba SS, Okongo MJ, Wagner H$\mathrm{U}$, Mulde D, et al. HIV-1 disease progression and AIDSdefining disorders in rural Uganda. Lancet 1997; 350: 245-50.

10 Macallan DC. Wasting in HIV infection and AIDS. Journal of Nutrition 1999; 129(Suppl. 1S): 238S-42S.

11 Dannhauser A, Van Staden AM, Van der Ryst E, Nel M, Marais N, Erasmus E, et al. Nutritional status of HIV-1 seropositive patients in the Free State Province of South Africa: anthropometric and dietary profile. European Journal of Clinical Nutrition 1999; 53: 165-73.

12 Romero-Alvira D, Roche E. The keys of oxidative stress in acquired immune deficiency syndrome apoptosis. Medical Hypotheses 1998; 51(2): 169-73.

13 Gramlich LM, Mascioli EA. Nutrition and HIV infection [Review]. Journal of Nutritional Biochemistry 1995; 6: 2-11.

14 Moscardini C, Tonger-Decker R, Ostroski MB. Nutritional needs in the AIDS patients. Recognizing and treating wasting syndrome. Advance for Nurse Practitioners 1997; 5(6): 34-7.

15 Casey KM. Malnutrition associated with HIV/AIDS. Part two: Assessment and interventions. Journal of the Association of Nurses in AIDS Care 1997; 8(5): 9-48.

16 World Health Organization/Food and Agriculture Organization of the United Nations (FAO). Living Well with HIV/AIDS. A Manual on Nutritional Care and Support for People living with HIV/AIDS. Rome: FAO, 2002; 1-97.

17 Vorster HH, Wissing MP, Venter CS, Kruger HS, Kruger A, Malan NT, et al. The impact of urbanisation on physical and mental health of South Africans in the Northwest Province of South Africa: the THUSA study. South African Journal of Science 2000; 96: 505-14.

18 Wissing M, Thekiso S, Stapelberg R, van Quickelberge L, Choabi P, Moroeng C, et al. The psychometric properties of scales measuring psychological well-being in an African group. Presented at International Africa Psychology Congress, Durban, South Africa, 18-23 July 1999.

19 MacIntyre UE, Venter CS, Vorster HH. A culture-sensitive quantitative food frequency questionnaire used in an African 
population: 1. Development and reproducibility. Public Health Nutrition 2000; 4(1): 53-62.

20 Kotler DP. Management of nutritional alterations and issues concerning quality of life. Journal of Acquired Immune Deficiency Syndromes and Human Retrovirology 1997; 16(Suppl. 1): S30-5.

21 Langenhoven M, Kruger M, Gouws E, Faber M. MRC Food Composition Tables, 3rd ed. Parow: Medical Research Council, 1991; 245.

22 Food and Nutrition Board, National Research Council, National Academy of Sciences. Recommended Dietary Allowances, 10th ed. Washington, DC: National Academy Press, 1989

23 James WPT. Policy and a prudent diet. In: Garrow JS, James WPT, Ralph A, eds. Human Nutrition and Dietetics, 9th ed. London: Churchill Livingstone, 1993; 767-75.
24 Van Staden AM, Barnard HC, Nel M, Attwood EM, Oosthuizen GM, Dannhauser A, et al. Nutritional status of HIV-1 seropositive patients in the Free State Province of South Africa. Central African Journal of Medicine 1998; 44(10): 246-50.

25 Constans J, Peuchant E, Pellegrin JL, Sergeant C, Hamon C, Duboury L, et al. Fatty acids and plasma antioxidants in HIVpositive patients: correlation with nutritional and immunological status. Clinical Biochemistry 1995; 28: 421-6.

26 Anes WA, Warrier R, Lybanon JI, Sharma R. Plasma nutritional changes antedate AIDS in hemophiliacs. Clinical Research 1989; 37: 467A.

27 Grunfeld C. Metabolic mechanisms for wasting in AIDS. In Proceedings of the Second Annual Nutrition and AIDS Conference, Stanford University, Palo Alto, CA, USA, 1989; 13-6. 